\title{
PROTOZOARIOS PARÁSITOS DE Musca domestica LINNAEUS (DIPTERA: MUSCIDAE) EN LIMA. DOS NUEVOS REGISTROS PARA EL PERÚ
}

\section{PROTOZOAN PARASITES OF Musca domestica LINNAEUS (DIPTERA: MUSCIDAE) FROM LIMA. TWO NEW RECORDS IN PERU}

\author{
Martín Cárdenas y Rosa Martínez :
}

\section{RESUMEN}

Et presente trabajo tuvo por objetivo contribuir al conocimiento de dos protozoarios: Herpetomonas muscarum (Leidy, 1856) Ken 1880 y Octosporea muscaedomesticae Flu, 1911, parásitos de Musca domestica. Ambos represeitan nuevos registros para el Perú.

Los promastigotes de $H$. muscarum observados son de mayor tamaño que los señalados por otros autores, pues miden hasta $36 \mu$ de longitud. O. muscadomesticae presenta formas alargadas y plasmodiales, siendo ligeramente màs grandes que las señaladas por Bulnheim \& Vàvra (1968). Se ha determinado un porcentaje significativo de $H$. muscarum $(54,0 \%)$ y 0 . muscadomesticae $(27,0 \%)$ en los lotes de $M$. domestica investigados.

Palabras clave: Herpetomonas muscarum, Octosporea muscaedomesticae, Musca domestica, parásitos.

\section{ABSTRACT}

The goal of this research was to increase the knowledge of two parasitic protozoa: Herpetomonas muscarum (Leidy, 1856) Ken, 1880 and Octosporea muscaedomesticae Flu, 1911, in Musca domestica. Both represent new records in Peru.

The $H$. muscarum leptomonad are $36 \mu$ long and are larger than the ones cited by other authors. $O$. muscadomesticae presents large and plasmodial forms slightly larger than the ones studied by Bulnheim \& Vávra (1998).

In the $M$. domestica group surveyed, we determined a significant percentage of $H$. muscarum $(54,0 \%)$ and $O$. muscadomesticae $(27,0 \%)$.

Key words: Herpetomonas muscarum, Octosporea muscaedomesticae, Musca domestica, parasites.

\section{INTRODUCCIÓN}

El presente trabajo tiene por finalidad contribuir al conocimiento de la fauna parasitaria propia de Musca domestica en el Perú.

La mosca doméstica es un insecto díptero que está muy asociado al hombre, algunos trabajos Jo relacionan con la transmisión mecánica du formas parasitarias patógenas para el

*Laboratorio de Parasitología General. Fac. Ciencias Biológicas. UNMSM. Av. Venezuela cuadra 34 s/n, Lima. E-mail: d190033@unmsm.edu.pe, jomarcs@mixmail.com ser humano (Coutinho et al., 1957; Guillén de Tantaleán et al, 1984; Keiding, 1987; Gamarra \& Ocampos. 1996; Custodio \& Murga, 1997; Manrique \& Delfín, 1997; Miranda \& Martínez, 1997). Sin embargo, en nuestro país no se han realizado estudios acerca de los protozoarios parásitos propios de este insecto.

En el ámbito internacional existen algunos aportes al respecto, siendo los más resaltantes los referentes al flagelado Herpetomonas muscarum y al microsporidio octosporea muscaedomesticae, parásitos del 


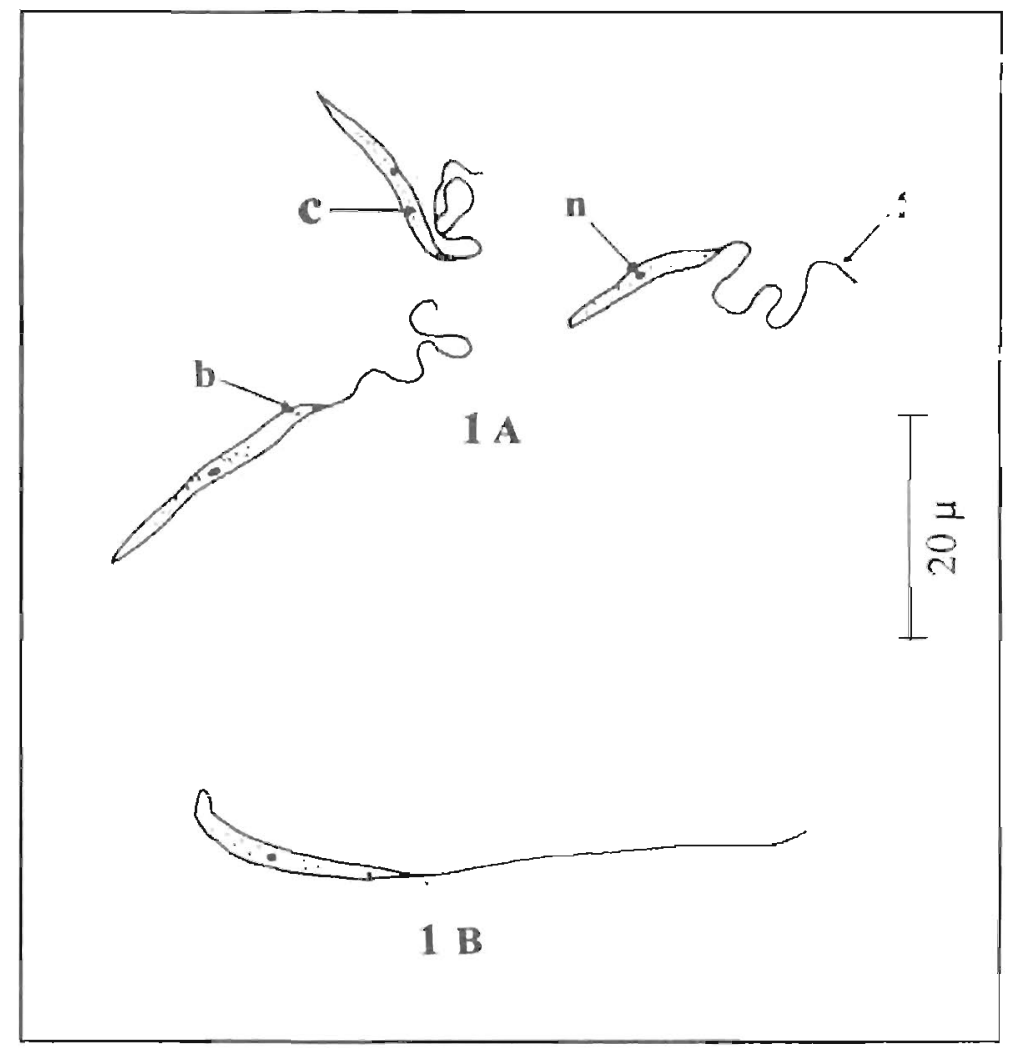

Figura 1. A y B. Formas típicas fusiformes de H. muscarum, donde "c" es el citoplasma, "n" el núcleo, "b" el blefaroplasto y "f" el flagelo.

tubo digestivo y el último también de los túbulos de malpighi de Musca domestica. (Kramer, 1963; Rogers \& Wallace, 1971; Ormieres et al., 1976; Vickerman, 1976; Wallace, 1976; Kudo, 1985).

\section{MATERIAL Y MÉTODOS}

Se colectaron 3014 "moscas" de la especie Musca domestica de los botaderos de basura formados en la vía pública de pueblos jóvenes de los distritos de Comas y de San Juan de Lurigancho, departamento de Lima, Perú, entre los meses de junio a diciembre de 1998. Este grupo de insectos correspondió a 100 lotes, constituidos por un número promedio de 30 especímenes.

La captura de las moscas se efectuó utilizando una red entomológica de tamaño mediano (aro de $15 \mathrm{~cm}$ de diámetro) confeccio- nada para tal fin. Luego los insectos fueron colocados en bolsas de polietileno, las que eran debidamente rotuladas con la procedencia y cantidad colectada.

Las moscas fueron sacrificadas con sobredosis de cloroformo. La disección de los especímenes se realizó sobre una lámina portaobjetos y los intestinos se colocaron en viales que contenían agua destilada estéril, se agitaron y se dejaron sedimentar. Parte del sedimento se fijó sobre una lámina portaobjetos con alcohol polivinílico (PVA). Posteriormente las muestras fijadas fueron coloreadas por el método Tricrómico de Gomori (Tantaleán, 1998). Adicionalmente, el intestino de uno o dos especímenes de cada lote se procesó para su observación directa con unas gotas de solución salina.

Para la identificación y estudio de los protozoarios parásitos de $M$. domestica se uti- 


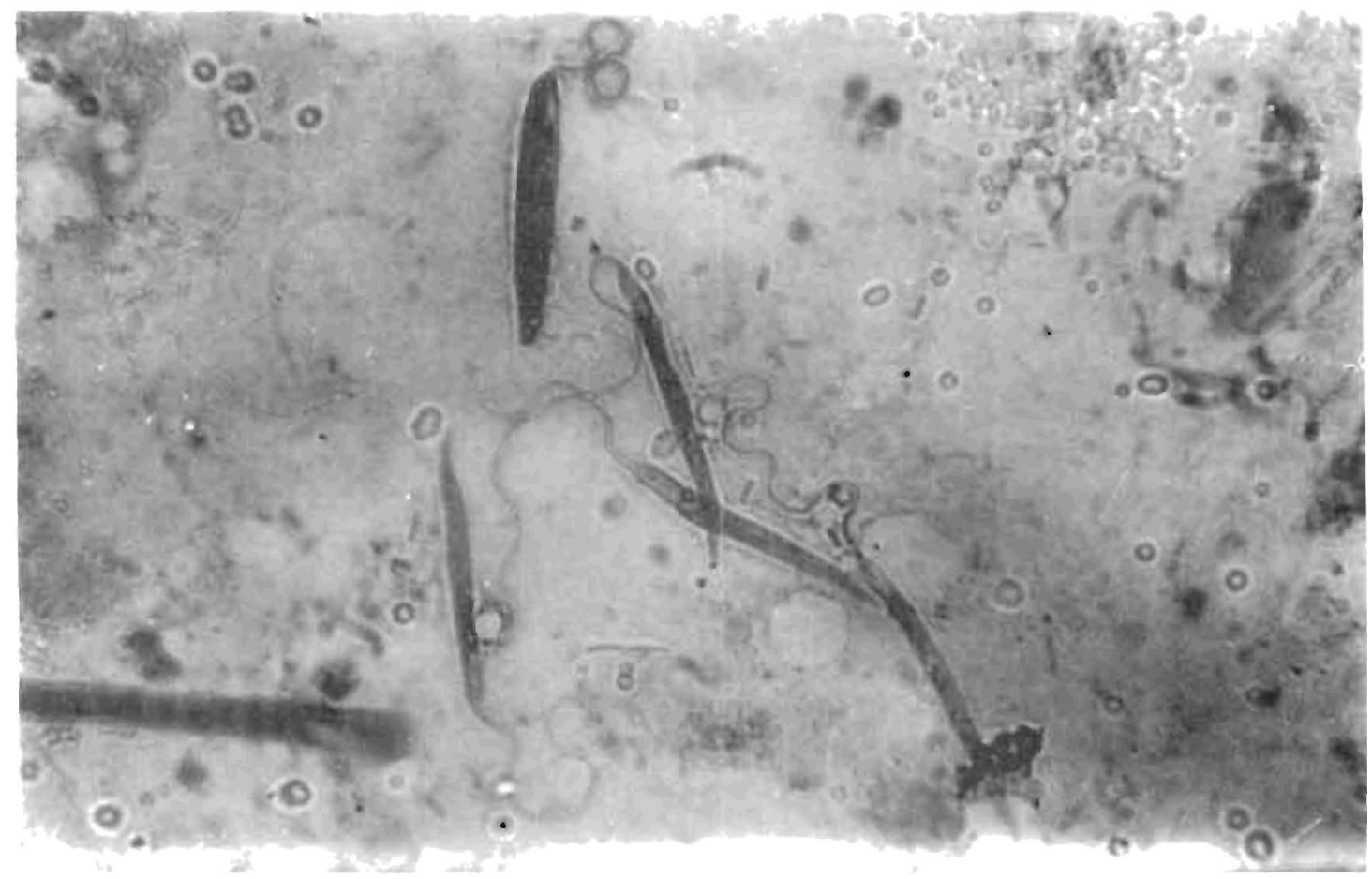

Figura 2. Promastigotes de H. muscarum vistos en el microscopio de contraste de fase (1000X).

lizaron como referencia los trabajos de Kramer (1963), Wallace (1963), Hoare \& Wallace (1966), Bulnheim \& Vávra (1968), Rogers \& Wallace (1971), Vickerman (1976), Ormieres et al. (1976) y Levine et al. (1980).

Los dibujos se hicieron utilizando una cámara lúcida y para las medidas un ocular micrométrico calibrado, ambos CARL ZEISS. Las medidas se expresan en micras, anotando primero el rango y luego el promedio entre paréntesis.

Las fotomicrografías fueron tomadas con un microscopio compuesto CARL ZEISS, con cámara fotográfica incorporada.

\section{RESULTADOS}

Se identificaron las siguientes especies:

Herpetomonas muscarum (Leidy, 1850) Ken, 1880

Posicióı taxonómica (según Levine et al., 1980):
Reino: Protista.

Subreino: Protozoa

Phylum: Sarcomastigophora

Subphylum: Mastigophora

Clase: Zoomastigophora

Orden. Kinetoplastida

Suborden: Trypanosomatina

Familia: Tripanosomatidae Género: Herpetomonas

Especie: H. muscarum

Descripción: Cuerpo alargado, fusiforme, algunas veces esbelta y con el extremo posterior truncado o puntiagudo. Presentan movimiento zigzagueante. El núcleo es central o ligeramente excéntrico. Poseen un flagelo libre que emerge por la abertura de un estrecho reservorio situado en la parte anterior del cuerpo, donde se ubica el blefaroplasto. La longitud del cuerpo es de 13,7 a $30,3(22,0)$ con un ancho corporal de 1,2 a 2,5 $(1,85)$. El flagelo mide de 36,0 a 44,2 $(40,1)$, casi el doble de la longitud del cuerpo (Figs. 1 y 2). 


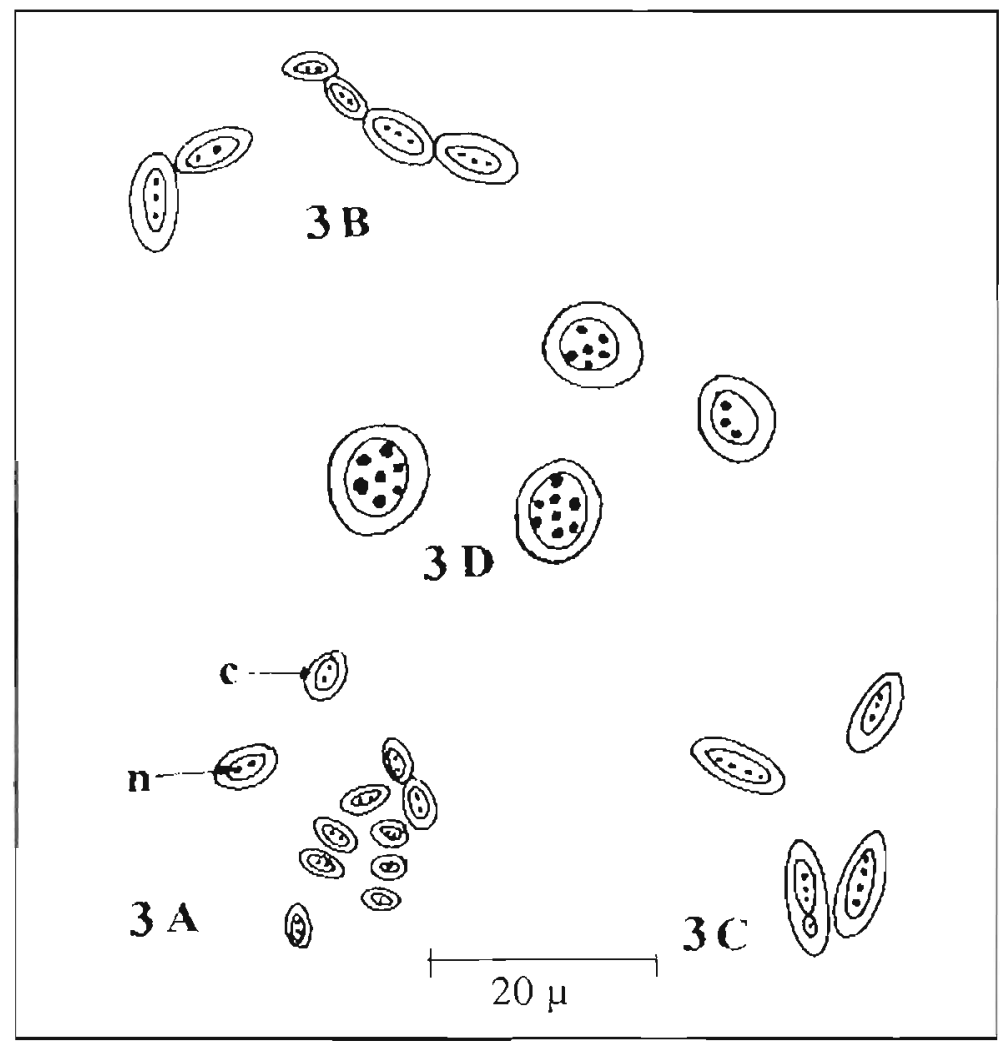

Figura 3. A, B, C y D. Diversas formas estructurales de $O$. muscadomesticae. A. Quistes binucleados: "n" núcleos y "c" envoltura. B. Formas alargadas con 2 a 4 núcleos. C. Formas alargadas con proyecciones citoplasmáticas a manera de yemas. D. Formas plasmodiales con 4 a 8 estructuras internas a manera de núcleos densos.

Hábitat: Tracto intestinal.

Uctosporea muscaedomesticae Flu, 1911

Posición taxonómica (según Levine et al., 1980):

Reino: Protista

Subreino: Protozoa

Phylum: Microspora

Clase: Microsporea

Orden: Microsporida

Género: Octosporea

Especie: O. muscaedomesticae

Descripción: Las esporas presentan forma cilíndrica, oval, alargada y ocasionalmente arqueada; generalmente son binucleadas. Miden de 2,5 a 6,0 $(4,25)$ de largo por 1,5 a $2,5(2,0)$ de ancho. Algunas veces se presen- $\tan$ formas acintadas con 4 a 8 núcleos y que pueden poseer protuberancias a manera de yemas en uno de sus extremos. Las formas acintadas miden de 6,6 a $12,0(9,3)$ de largo por 2,0 a $2,5(2,25)$ de ancho. Se observan además formas plasmodiales de 6 a $7,5(6,75)$ de diámetro y que poseen 4 a 8 formaciones internas a manera de núcleos densos. Todos los estadios observados están rodeados por una envoltura a manera de cápsula de 0,8 a 2,5 (1,65) de grosor (Figs. 3 y 4).

Hábitat: Epitelio intestinal.

De los 100 lotes investigados 80 (80\%) resultaron positivos a la presencia de alguna forma parasitaria en general. Con respecto al porcentaje de lotes positivos por tipo de pro- 


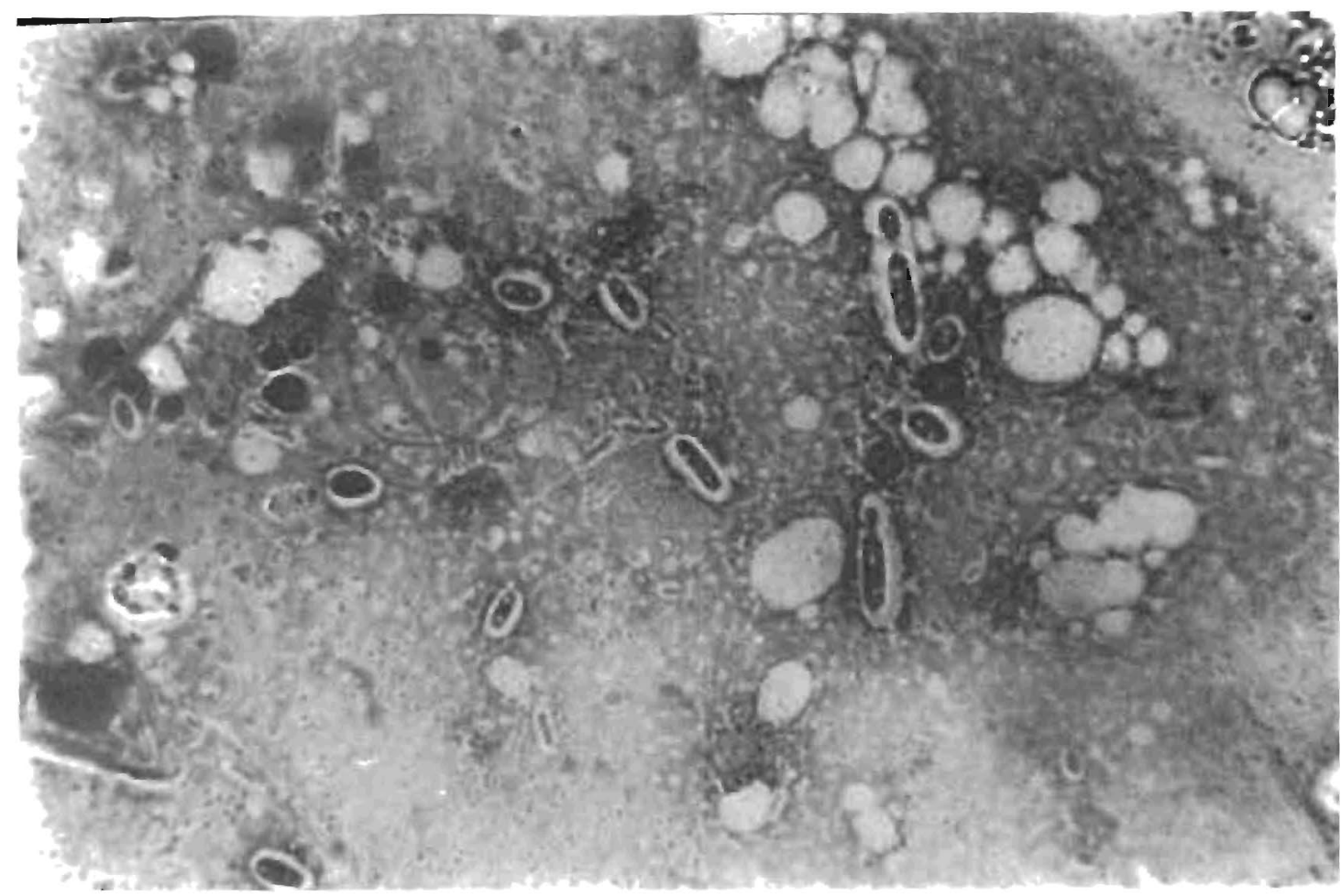

Figura 4. Esporas de O. muscadomesticae. Coloración Tricrómica de Gomori (1000X).

tozoario parásito de $M$. domestica, en 54 lotes $(54 \%)$ encontramos a $H$. muscarum, y en 27 lotes $(27 \%)$ a $O$. muscaedomesticae (Tabla 1).

\section{DISCUSIÓN}

En el Perú no se conoce documento alguno que muncione a los protozoarios parásitos propios de $M$. domestica, cuyo conocimiento básico es de suma importancia, porque son indispensables para dar soporte técnico y planificar las estrategias de control de las poblaciones de este insecto y de otros dípteros.

El presente estudio constituye el primero que trata de los protozoarios parásitos propios de $M$. domestica. Del mismo modo es la primera investigación que se realiza en el distrito de Comas que, al igual que San Juan de Lurigancho, presenta condiciones ecológicas adecuadas para la proliferación de $M$. domeslica, como son un saneamiento inadecuado y la gran cantidad de botaderos de basura (acumulación de residuos sólidos domiciliarios) formados en la vía pública.

Dos protozoarios resultaron ser los primeros hallazgos para el Perú: Herpetomonas muscarum (Leidy, 1856) Ken, 1880 y Octosporea muscaedomesticae Flu, 1911, ambos parásitos nàturales de $M$ domestica (Rogers \& Wallace, 1971; Ormieres et al., 1976). El primero es el protozoo más conocido de este insecto, por ser el más frecuentemente nombrado en las referencias consultadas, mientras que los aportes sobre $O$. muscaedomesticae son escasos.

H. muscarum es la especie más común que parasita el tubo digestivo de la "mosca doméstica" y otros dípteros. La posición taxonómica de este flagelado está muy bien esclarecida y la clasificación que presentamos sigue la de Levine et al. (1980). Las características más subresalientes de los 
Tabla 1. Porcentaje de protozoarios parásitos de Musca domestica de los distritos de Comas y San Juan de Lurigancho (Lima).

\begin{tabular}{|c|c|c|c|c|c|}
\hline \multirow[b]{2}{*}{ LOCALIDAD DE CAPTURA } & \multirow[b]{2}{*}{$\begin{array}{l}\text { LOTES } \\
\text { EXAMINADOS }\end{array}$} & \multicolumn{2}{|c|}{$H_{\text {. muscarum }}$} & \multicolumn{2}{|c|}{ O. muscadomesticae } \\
\hline & & POSITIVOS & $\%$ & POSITIVOS & $\%$ \\
\hline \multicolumn{6}{|l|}{ COMAS } \\
\hline COLLIQUE & 7 & 3 & 42.9 & 4 & 57,1 \\
\hline AÑO NUEVO & 8 & 3 & 37.5 & 1 & 12,5 \\
\hline SAN JUAN BAUTISTA & 8 & 5 & 62,5 & 1 & 12,5 \\
\hline SINCHI ROCA & 10 & 7 & 70,0 & 2 & 20.0 \\
\hline LA PASCANA & 9 & - & - & 3 & 33,3 \\
\hline LA MEACED & 8 & - & - & 2 & 25,0 \\
\hline \multicolumn{6}{|l|}{ SAN JUAN DE LURIGANCHO } \\
\hline HUÁSCAR I & 13 & 10 & 76.9 & 3 & 23,1 \\
\hline HUÁSCAR \| & 8 & 4 & 50,0 & 1 & 12,5 \\
\hline 10 DE FEBRERO & 7 & 6 & 85,7 & 2 & 28,5 \\
\hline CANTO REY & 6 & 4 & 66.7 & 3 & 50,0 \\
\hline LAS FLORES & 8 & 6 & 75,0 & 3 & 37,5 \\
\hline JESUंS OROPEZA & 8 & 6 & 75,0 & 2 & 25,0 \\
\hline TOTA: & 100 & 54 & 54,0 & 27 & 27,0 \\
\hline
\end{tabular}

Kinetoplastida de artrópodos, con especial referencia al género Herperomonas, son detalladas por Vickerman (1976) y Wallace (1976).

Según la descripción de algunos autores, las formas de $H$. muscarum provenientes de $M$. domestica miden de 10 a 25 (Rogers \& Wallace, 1971), 12 a 18 por 2 (Olsen; 1977), y de 30 por 2 a 3 (Kudo, 1985). En las muestras analizadas en el presente trabajo se observaron organismos que llegan a medir hasta 36 de longitud, siendo de mayor tamaño que las anteriormente mencionadas.

La característica más sobresaliente del género Herpetomonas es la ocurrencia en simultáneo de los estadios promastigote y opistomastigote durante su ciclo de vida (Hoare \& Wallace, 1966; Rogers \& Wallace, 1971; Vickerman, 1976; y Wallace, 1976). En la presente investigación, por el tamaño y las características morfológicas observadas, la mayoría corresponden al primer estadio.

La bibliografía revisada no menciona la prevalencia de este flagelado en las moscas a las que afecta; sin embargo, de acuerdo a nuestros estudios, ésta es elevada en la ciudad de
Lima, pues se la observó en 4 de 6 localidades investigadas y en el 54,0\% del total de los lotes investigados (Tabla 1). El elevado porcentaje de lotes positivos con este parásito nos indicaria que su presencia es normal en las moscas de las localidades investigadas y sobre todo en aquellos lugares donde las condiciones del medio ambiente son propicias para el desarrollo de este flagelado y de sus hospedadores.

Es importante el estudio de estos Tripanosomatidae porque permitiria establecer posibles aplicaciones en inmunología o la utilización de los flagelados como modelo en estudios de diferenciación celular, quimioterapia y simbiosis. La estrecha relación que existe entre Herperomonas, Tryparosoma y Leishmania sugiere que podrían estar emparentados inmunológicamente (Wallace; 1976).

Con relación al microsporidio Octosporea muscaedomesticae, su posición taxonómica aún no esta muy bien definida y la clasificación que presentamos sigue a Levine et al. (1980), Bulnheim \& Vávra (1968) y Ormieres et al. (1976). 
Existe una discusión en torno a la posición taxonómica de $O$. muscaedomesticae para ubicarlo dentro del suborden Pansporoblastina o Apansporoblastina, es decir si presenta o no membrana pansporoblástica. De acuerdo a Bulnheim \& Vávra (1968), quienes mencionaron que en $O$. gammarus las esporas se originan por el rompimiento de un pansporoblasto, se ubicaría en el subo:den Pansporoblastina. Del mismo modo, Ormieres et al. (1976), al estudiar especímenes de $O$. muscaedomesticae, señalaron la presencia de estructuras semejantes a una membrana y propusieron que podría incluirse al género Octosporea en el suborden Pansporoblastina. Sin embargo, al no contar con más datos, nosotros nos limitamos a dar sólo la clasificación anotada.

Según algunos autores las esporas maduras de 0 . muscaedomesticae son binucleadas y miden 5,5 a 7,5 por 1,8 a 2,5 (Kramer, 1963) $y$ de 5,5 a 6 por 1,5 (Ormieres et al., 1976); nosotros encontramos esporas que midieron de 2,5 a 6,0 de largo por 2 de ancho, siendo estas medidas bastantes coincidentes con las halladas por los investigadores referidos.

En cuanto a la morfología de las esporas, observamos típicas formas ovaladas además de esporas alargadas y con proyecciones citoplasmáticas que llegan a medir hasta 12 de largo; así también formas redondeadas (plasmodiales) de 6 a 7,5 de diámetro, siendo ambas ligeramente más grandes que las señaladas por Bulnheim \& Vávra (1968), quienes de igual manera observaron estas formas en $O$ effeminans. Todos los estadios están rodeados por una especie de envoltura que podría ser una membrana o el producto de la reacción del hospedero a la infección. Las diversas formas estudiadas confirman lo expresado por estos investigadores, quienes señalaron que a lo largo del ciclo de vida de esta especie de protozoario se presentan múltiples formas estructurales de diferentes tamaños.

Al igual que $H$. muscarum, el porcentaje significativo de lotes positivos con $O$. muscaedomesticae nos demuestra que en nuestro medio las condiciones son propicias para su desarrollo y que podría tener una prevalencia muy manifiesta en las localidades estudiadas. Nosotros encontramos lotes positivos en todas las localidades, alcanzando el $27.0 \%$ de los lotes investigados (Tabla 1). Las diferencias que podrían existir en el tamaño de las esporas se deberían a las variaciones ambientales exigidas por los hospedadores o a mecanismos de especiación del Phylum (García, 1992).

Tanto $H$. muscarum como $O$. muscaedomesticae son protozoos que no afectan al hombre; sin embargo, algunos géneros de microsporidios son causantes de infecciones serias en personas inmunosuprimidas (Bryan et al., 1991). Del mismo modo algunos géneros son patógenos y producen la muerte de un gran número de insectos (Olsen, 1977); de aquí se desprende la importancia del estudio de estos organismos cuyos hospedadores están estrechamente relacionados con el hombre.

\section{Agradecimientos}

Al Dr. Manuel Tanılaleán V. por las sugerencias y la revisión del manuscrito.

\section{LITERATURA CITADA}

Bryan, R.; Cali, A.: Owen, R. \& H. Spencer. 1991. Microsporidia: Opportunistic Pathogens in Patients with AlDS. Progress in Clinical Parasitology 2: 1-26.

Bulnheim, H. \& J. Vávra. 1968. Infection by the Microsporidian Octosporea effeminans sp. n., $\&$ its Sex Determining Influence in the Amphipod Gammarus diebeni. J. Parasitol. 54: $241-248$.

Coutinho, J.; Taunay. A. y L. Penna. 1957. Importancia da Musca domestica como Vector de Agentes Patogénicos para o Homen. Rev. Inst. Adolfo Lutz 17: 5-23.

Custodio, M. y M. N. Murga. 1997. Enteroparásitos Transportados por Musca domestica, en Moche. Trujillo-Perú. Libro de Resúmenes del II Congreso Peruano de ParasitologíaArequipa, pág. 117.

Gamarra, M. y S. Ocampos. 1996. Musca domestica y Blatridae como Vectores Mecánicos de Enteroparásitos en Pueblos Jóvenes del Dis- 
trito de Monsefú, dpto. de Lambayeque 1995. Tesis Biología. Univ. Nac. "Pedro Ruiz GaIlo", Lambayeque, 59 pp.

García, J. 1992. Patógenos de Simulidos Neotropicales (Diptera: Simuliidae): Amblyospora bracteala (Strickland, 1913) (Microspora: Thelohaniidae). Rev. Soc. Ent. Argent. 50: 3-8.

Guillén de Tantaleán, Z.; MartÍnez, R.; Del Águila, A. y R. Cusi. 1984. "Moscas" y "cucarachas" como Vectores de Parasitosis en el Pueblo joven 14,5 Hectáreas-Callao. Bol. Trabajo Extramural. Programa Académico de Medicina Humana, UNMSM 1: 13-16.

Hoare, C. \& F. Wallace. 1966. Developmental Stages of Trypanosomatid Flagellates: a New Terminology. Nature 212: 1385-1386.

Keiding, J. 1987. La Mosca Doméstica: Biología y Control. Documento de la Organización Mundial de la Salud. UMS/VBC/86.937,69 pp.

Kramer, P. 1963. A Microsporidian of the Genus Octosporea a Parasite of Phormia regina (Meigen) \& Callitroga macellaria (Fabricius) (Diptera: Calliphoridae). Proceedings of the First International Congress on Protozoology, Prague. Progress in Protozoology: 495-496.

Kudo, R. R. 1985. Protozoología. 8." impresión. Editorial Continental. S. A., México D. F.

Levine, N.; Corliss, J.; Cox, F.; Deroux, G.; Grain, J.; Honingberg, B.; Leedale, G.; Loeblick, A.: Lom, J.; Lynn, D.; Merinfeld, E.; Page, F.; Poljansky, G.; Sprague, V.; Vavra..J. \& F. Wallace. 1980. A Newly Revised Classification of the Protozoa. J. Protozool. 27: $37-58$.
Manrique, P. y H. Delfín. 1997. Importancia de las Moscas como Vectores Potenciales de Enfermedades Diarreicas en Humanos. Rev. Biomédica 8: 163-170.

Miranda, E. y R. Martínez. 1997. Estudio Preliminar sobre la Importancia de las Moscas como Transmisoras Mecánicas de Ooquistes de Cryptosporidium sp. Libro de Resúmenes de la VI Reunión Científica ICBAR, p. 84.

Olsen, O. 1977. Parasitología animal. Vol. I, Primera Edición, Barcelona, 284 pp.

Ormieres, R.; Baudouin. J.; Brugerolle, G. et R. Pralavorio. 1976. Ultraestructure de Quelques Stades de la Microsporidie Octosporea muscaedomesticae Flu, Parasite de Ceratitis capilata (Wiedemann) (Diptera, Trypetidae). J. Protozool. 23: 320-328.

Rogers, W. F. Wallace. 1971. Two New Subspecies of Herpetomonas muscarum (Leidy, 1856) Ken. 1880. J. Protozool. 18: 645-649.

'Tantaleán, M. 1998. Coloración de Protozoarios del Tubo Digestivo Para Preparaciones permanentes, $15 \mathrm{pp}$.

Vickerman, K. 1976. In "Biology of the Kinetoplastida". W. H. R. Lumsden and D. A. Evans Eds. I: 1 34.

Wallace, F. 1963. Criteria for the Differentiation of Genera among the Trypanosomatid Parasites of Insects. Proceedings of the First International Congress on protozoology, Prague. Progress in protozoology: 70-74.

Wallace, F. 1976. In "Biology of the Kinetoplastida" W.H:R. Lumsden and D. A. Evans Eds. IY: 213-240. 